\title{
Estudio de las fases de juego a través del análisis del entrenamiento deportivo en categoría minibasket
}

\author{
Study of the phases of game through the analysis \\ of sport training in U'12 categories
}

\section{Estudo das fases do jogo através da analises do treinamento esportivo na categoria de mini - basquete}

\author{
Cañadas, M. ${ }^{1}$, Ibáńez, S. J. ${ }^{2}$, García, J. ${ }^{2}$, Parejo, I. ${ }^{3}$, y Feu, $S .{ }^{2}$ \\ ${ }^{1}$ Universidad de Murcia. España. ${ }^{2}$ Universidad de Extremadura. España. \\ ${ }^{3}$ Fundación Valhondo Calaff
}

\section{RESUMEN}

El objeto de este estudio fue analizar la planificación de las fases de juego que realiza un entrenador durante el entrenamiento. La muestra la constituyen la totalidad de las tareas de entrenamiento de dos temporadas deportivas $(n=846)$. Las variables de estudio fueron las fases de juego, los medios de entrenamiento y las situaciones de juego. Se analizaron las diferencias en el tratamiento de las variables en las dos temporadas y la relación entre las variables. Los resultados ponen de manifiesto el predominio de la fase de ataque sobre la de defensa. El tratamiento de los contenidos ofensivos se plantea con la utilización de ejercicios y con situaciones de juegos sin oposición (1x0). Para el trabajo defensivo se opta por los juegos y las situaciones de $1 \times 1$.

PALABRAS CLAVE Fases de juego, Iniciación deportiva, Entrenamiento Deportivo, Entrenador, Baloncesto.

ABSTRACT

The aim of this study is to analyze the planning phases of play that are carried out by a coach during training. The sample is made up of all training tasks of two sport seasons ( $n=846)$. The variables studied were the phases of play, media training and game situations. The differences in the treatment of variables in the two seasons and the relationship between the variables were analyzed. The results show the predominance of the attack phase over the defense. The treatment of offensive contents arise with the use of exercises and game situations without opposition $(1 \mathrm{x} 0)$. For the defensive training is chosen the games and situations of $1 \times 1$.

KEY WORDS Phases of game, Sport initiation, Coaching, Coach, Basketball.

RESUMO

O objetivo desse estudo foi de analisar a planificação das fases de jogo que realiza um treinador durante o treinamento. A mostra constituiu de uma totalidade de tarefas de treinamento de duas temporadas esportivas $(n=846)$. As variáveis do estudo foram às fases do jogo, os meios de treinamento e as situaçóes de jogo. Foram analisadas as diferenças no tratamento das variáveis nas duas temporadas e a relação entre as variáveis. Os resultados manifestaram um predomínio da fase de ataque sobre a defesa. O tratamento dos conteúdos ofensivos se apresenta com a utilizaçáo de exercícios e com situações de jogos sem oposição (1x0). Para o trabalho defensivo opta-se pelos jogos e situaçóes de $1 \times 1$.

PALAVRAS CHAVE: fase de jogo, iniciação esportiva, treinamento esportivo, treinador, basquete.

\section{Introducción}

Los estudios sobre el talento deportivo pretenden identificar cuáles son los aspectos que determinan la excelencia en el deporte. La detección temprana del talento no asegura el éxito puesto que el desarrollo de los deportistas es un proceso que se ve influido por multitud de factores (Sáenz-López, Ibáñez, Giménez, Sierra, y Sánchez, 2005). Este tipo de trabajos ponen de manifiesto la importancia del entrenamiento deporti-

Dirección de contacto: María Cañadas Alonso

Facultad de Ciencias del Deporte. Universidad de Murcia. C/ Argentina $\mathrm{S} / \mathrm{N}$.

30.720 Santiago de la Ribera. Murcia. España

Tlf.657518357.e-mail: mariacanadas@um.es vo y de la idoneidad del proceso formativo para un adecuado desarrollo y perfeccionamiento en jugadores y jugadoras de baloncesto. La realidad requiere analizar el proceso de entrenamiento deportivo para su optimización, en concreto en las etapas formativas iniciales que suponen la base de las siguientes pudiendo determinar resultados futuros. Es necesario conocer con precisión cómo se desarrolla el entrenamiento deportivo (Ibáńez, 2008).

En la línea de analizar el proceso de entrenamiento, en un deporte como el baloncesto, surgen trabajos que se centran en estudiar la propia situación de entrenamiento, en concreto las tareas de entrenamiento. La actividad o tarea propuesta supone la unidad más concreta de la programación deporti- 
va. Sáenz-López (2009) apunta que la tarea es el nivel de la planificación al que se le dedica menos atención en las publicaciones de esta temática.

Movidos por la importancia de saber cómo son las situaciones de entrenamiento propuestas para la enseńanza del baloncesto en categorías de iniciación, se han desarrollado estudios sobre el tipo de tareas de entrenamiento que se plantea para el entrenamiento de deportes colectivos. A modo de ejemplo trabajos como los de Cañadas y García (2005), Moreira, Pinto y Graça (2007), Saad y Nascimento (2007), Cañadas, Parejo, Ibáñez, García y Feu (2009) y Cañadas e Ibáñez (2010).

Han sido varias las perspectivas desde las que se han abordado el análisis de las tareas de entrenamiento diferenciando entre un análisis cuantitativo o cualitativo. Las sesiones de entrenamiento pueden ser caracterizadas por aspectos cuantitativos o cualitativos. La carga o volumen de entrenamiento hace referencia a la dimensión cuantitativa, mientras que aspectos relacionados con la estructura o las características de las propias tareas se referirían a la parte cualitativa (Sampedro, 1999). Sin embargo, en la actualidad esta barrera es cada vez más fina, de forma que ambos tipos de análisis tienden a combinarse. Un ejemplo de ello son los estudios de Refoyo, Sampedro, Lorenzo y del Campo (2009) en el que cuantifican una serie de valores fisiológicos y los relacionan con la respuesta técnico-táctica que generan las tareas en los jugadores de baloncesto. Un aspecto como la carga del entrenamiento ha dejado de tener un carácter eminentemente cuantitativo, ámbito fisiológico, para ser analizado desde una perspectiva cualitativa. Trabajos como el de García, Parejo y Cañadas (2010) o la propuesta de Cárdenas (2009) evalúan la "carga" de una actividad en función de su dificultad a nivel perceptivo, decisional o de ejecución.

Desde una perspectiva eminentemente cualitativa, encontramos trabajos que se centran en analizar las características de las tareas de entrenamiento con el objeto de saber cómo es el entrenamiento deportivo diseńado en una temporada, para un equipo de una categoría o nivel determinado, etc.

Son varias las características desde las que han sido definidas las tareas de entrenamiento. Desde una perspectiva praxiológica encontramos trabajos como los de Martínez de Santos (2004) que basados en la lógica interna de un deporte como el fútbol caracteriza las diferentes tareas de entrenamiento atendiendo a parámetros praxiológicos ó el de Jiménez (2000) en dos deportes colectivos como el balonmano y el fútbol sala. Otros criterios son utilizados por autores como Rink ó Ticó, basados en la semejanza que poseen las tareas con la situación de juego real. Ibáńez (2008) propone una serie de elementos que definen las tareas denominándolos como variables pedagógicas del entrenamiento. Tal y como muestran los estudios realizados por Cañadas et al. (2009) y Cañadas e Ibáñez (2010), el análisis de estas variables permite describir cómo se está desarrollando el entrenamiento deportivo desde el punto de vista técnico-táctico, conociendo por ejemplo el tipo de medios de entrenamiento que se utilizan, situaciones de juego o cómo son organizados los contenidos de baloncesto a lo largo de la temporada deportiva.

El entrenamiento de baloncesto, y en concreto la forma en que se planifica el entrenamiento deportivo de este deporte, puede ser analizado a través del estudio de las variables pedagógicas que definen el entrenamiento deportivo (Cañadas et al., 2009; Ibáñez, 2007, 2008, 2009). Una de esas variables pedagógicas es la fase de juego. Desde una perspectiva metodológica, el baloncesto es un juego donde predominan las acciones colectivas en su doble faceta ofensiva y defensiva. Para la enseñanza de los deportes de equipo es necesario conocer la estructura del juego y cumplir los principios que lleven a una adecuada enseńanza/entrenamiento del deporte (Sampedro, 1999).

En la literatura se encuentran aportaciones teóricas de cómo se debe plantear el trabajo de las fases de juego. A modo de ejemplo citar las propuestas de Giménez y Sáenz-López (2007), Ibáñez (2000, 2008), Sáenz-López (2009) entre otros.

Con el objeto de profundizar en el proceso de entrenamiento deportivo en este ámbito también se han desarrollado otro tipo de trabajos que se centran en conocer la opinión sobre los distintos contenidos ofensivos y defensivos de baloncesto que tienen entrenadores (Cañadas, Ibáñez, García, y Parejo, 2009; Leite, 2009; Ortega, 2010; Salado, Bazaco, Ortega, y Gómez, 2011) y jugadores (Palao, Ortega, y Olmedilla, 2007). Este tipo de estudios ofrecen la posibilidad de conocer las opiniones de personas que intervienen en el entrenamiento deportivo aportando una visión mucho más global del proceso (Leite, 2009). Sin embargo, se precisa de un conocimiento de la realidad que informe sobre la forma en que se desarrolla el entrenamiento deportivo, que conteste a las múltiples preguntas que aún existen sobre el entrenamiento deportivo y que aporte un conocimiento que pueda ser aplicado a la realidad más cercana como es la propia sesión de entrenamiento (Cañadas e Ibáñez, 2010).

La planificación y registro del entrenamiento es un procedimiento básico para conocer objetivamente cómo se produce el entrenamiento en deportes colectivos (Cañadas e Ibáñez, 2010; Ibáńez, 2008). Autores como Sampedro (1999) y Sáenz-López y Giménez (2006), señalan la importancia de planificar el trabajo a desarrollar apuntando que son varias las ventajas que aporta este procedimiento. La planificación y control del entrenamiento son procesos que favorecen la optimización del proceso de entrenamiento y por ende el proceso formativo de los jugadores de baloncesto (Ibáńez, 2007, 2008). Para facilitar el proceso de planificación y posterior control de las sesiones de entrenamiento programadas, se han diseñado diferentes aplicaciones. Entre ellas, se encuentra la herramienta informática $P y C$ Basket 2.0, programa creado 
para la planificación y control del entrenamiento deportivo en baloncesto (Ibáñez, Macías, Pérez, y Feu, 2001).

La descripción y registro del entrenamiento deportivo forma parte del ámbito a través del cuál se registran los comportamientos, sucesos o acciones que se producen en una dimensión para proceder a su análisis. Una dimensión que en este caso se trata del entrenamiento, y de forma más concreta de la tarea de entrenamiento. Entre las técnicas que se han utilizado para registrar esta dimensión se puede distinguir entre aquellos trabajos en los que es otro agente externo (investigador) el encargado del propio registro o los estudios en los que esta labor queda relegada al responsable de este suceso o comportamiento de estudio. En el caso que nos ocupa, el análisis de las tareas de entrenamiento se puede distinguir entre trabajos en los que un agente externo registra las características de cada tarea de entrenamiento, como ejemplo estudios como los de Graça (1997) o Lemos (2005), u otros en los que es el propio entrenador el encargado de este procedimiento, trabajos como los de Cañadas, Parejo, et al. (2009) y Cañadas e Ibáńez (2010). En cualquier de los casos es preciso asegurar la calidad del dato registrado. Resaltamos trabajos como los de Castellano, Hernández, Gómez de Segura, Fontetxa y Bueno (2000) y Gorospe, Hernández, Anguera y Martínez de Santos (2004), como referencia de los procedimientos utilizados para asegurar la calidad del dato entidad de análisis.

El entrenador es el principal responsable del proceso de entrenamiento. Sobre él recae la responsabilidad de la planificación, el desarrollo y el control del entrenamiento deportivo (Ibáñez, 2008, 2009). De ahí que, a menudo el análisis de la planificación y del entrenamiento deportivo se asocie al estudio de la figura del entrenador. En esta línea encontramos trabajos como los de Feu (2006) e Ibáńez $(2007,2009)$ en los que se hace referencia a "modelos de entrenador" identificando lo que implica estos tipos de perfil para la planificación del proceso de entrenamiento. Con estos y otros trabajos en esta línea se pretende identificar la forma en que un entrenador diseña el entrenamiento deportivo analizando su relación con otros elementos como la categoría del equipo u otros más propios del entrenador como su experiencia o formación.

Centrando el objeto de estudio en el propio proceso de entrenamiento deportivo, este trabajo persigue conocer cómo se plantea, en concreto, el tratamiento de las fases de juego en el entrenamiento de baloncesto en categorías de iniciación diseñado por un entrenador para dos equipos de minibasket.

\section{Método}

El diseño de este estudio se encuadra dentro de los calificados como mixtos al utilizar diferentes metodologías para su desarrollo. Por un lado, desde la perspectiva del análisis de un caso concreto, entrenador que diseña los entrenamientos de dos equipos minibasket, se trata de un estudio empírico cualitativo que utiliza una metodología de estudio de caso único (Montero y León, 2007). Por otro lado, considerando el análisis de las tareas, y estas como muestra del estudio, y basados en el procedimiento llevado a cabo para el registro y análisis de este tipo de datos, este trabajo se clasifica como un estudio empírico con metodología cuantitativa (Montero y León, 2007) y, de forma específica, se trata de un estudio descriptivo mediante un código arbitrario de registro.

\section{Muestra}

La muestra del estudio la conforman, las tareas de entrenamiento planificadas para dos equipos de categoría minibasket diferentes. Para el estudio se recogieron la totalidad de las sesiones de entrenamiento de la temporada 1, 80 sesiones ( 452 tareas), y de la temporada 2, 74 sesiones (394 tareas). La temporada 1 y 2 se suceden en el tiempo existiendo un intervalo de un año entre ambas en el que el entrenador no desarrolló labores como primer entrenador y, por lo tanto, no se registraron datos durante esta temporada. En total se analizaron 846 tareas de entrenamiento entre las dos temporadas.

El entrenador de baloncesto encargado del diseño y registro de las tareas de entrenamiento también forma parte de la muestra del estudio. Este entrenador forma parte de un programa de formación de entrenadores dirigido por el grupo de investigación que ha llevado a cabo este trabajo. Este programa formativo inculca una metodología alternativa, abordando la enseńanza de este deporte desde una perspectiva comprensiva y basado en una metodología constructivista. El entrenador de estudio se inicia en este programa de formación antes de comenzar la temporada deportiva de estudio.

\section{Variables}

La variable del estudio es la fase de juego. Es una de las variables pedagógicas que describen el entrenamiento deportivo (Ibáńez, 2008). Las categorías de esta variable son: fase de ataque (tarea de entrenamiento planificada para trabajar la fase ofensiva o, predominantemente, contenidos ofensivos), fase de defensa (tarea para trabajar la fase defensiva del juego o, predominantemente, contenidos defensivos) y fase mixta (tarea en la que se concede la misma importancia al trabajo de los fundamentos ofensivos como los defensivos).

Para profundizar en el análisis de esta variable, se estudia su relación con otras dos variables pedagógicas como son: la situación de juego y los medios de entrenamiento. La situación de juego representa el número de jugadores que participan y la forma en que se relacionan en las tarea y los medios de entrenamiento se refieren al tipo de actividad planteada en la tarea de entrenamiento basada en la clasificación establecida por Ibáñez, Parra y Asensio (1999) (Ibáñez, 2008). 


\section{Instrumentos}

Para el registro de las sesiones de entrenamiento planificadas, se utilizó el programa informático PyC Basket 2.0, creado para la Planificación y el Control del entrenamiento deportivo (Ibáñez et al., 2001). El Pyc Basket ha sido confeccionado basado en las estructuras que definen el entrenamiento deportivo, en su sentido global, y de forma específica respecto a los elementos del entrenamiento en baloncesto definidos por expertos en el ámbito e incorporado en forma de categorías al software de registro.

Este instrumento también ha sido aplicado al baloncesto en trabajos como los de Cañadas, Parejo, et al. (2009) y Cañadas e Ibáñez (2010) entre otros.

\section{Procedimiento}

En primer lugar se procedió a la formación y entrenamiento del entrenador, encargado del registro de las tareas, y de los evaluadores externos, participantes en el procedimiento para calcular la calidad del dato registrado (Gorospe et al., 2005). Estas sesiones de formación y entrenamiento van destinadas al conocimiento de los criterios a tener en cuenta a la hora de clasificar cada una de las tareas de entrenamiento y sobre la utilización del programa informático PyC Basket 2.0.

Fueron dos los evaluadores externos que participaron en este proceso. Las características de los mismos aparecen en la siguiente tabla.

Tabla 1. Características de los dos evaluadores externos participantes en el procedimiento para el cálculo de la calidad de los datos.

\begin{tabular}{lcc}
\hline CARACTERÍSTICAS & E1 & E2 \\
\hline Titulación académica relacionada & $\mathrm{X}$ & $\mathrm{X}$ \\
Titulación federativa & $\mathrm{X}$ & $\mathrm{X}$ \\
Profesor universidad & $\mathrm{X}$ & $\mathrm{X}$ \\
Doctor & $\mathrm{X}$ & $\mathrm{X}$
\end{tabular}

Tabla 2. Resultados de la prueba de Pearson para el cálculo de la calidad de los datos.

Tabla 2. Resultados de la prueba de Pearson para el cálculo de la calidad de los datos.
\begin{tabular}{lllll}
\hline \multicolumn{1}{l}{ Variables } & Medios de entrenamiento & Situación de juego & Fase de juego \\
\hline A1 y A2 (intra) & .61 & .93 & .79 \\
B1 y B2 (intra) & .83 & .93 & .79 \\
C1 y C2 (intra) & .72 & .93 & .82 \\
A1 y B1 (inter) & .61 & .73 & .79 \\
A1 y C1 (inter) & .75 & .82 & .82 \\
B1 y C1 (inter) & .84 & .90 & .96 \\
A2 y B2 (inter) & .71 & .73 & .79 \\
A2 y C2 (inter) & .70 & .84 & .86 \\
B2 y C2 (inter) & .97 & .89 & .97 \\
\hline
\end{tabular}

Para estimar la validez y fiabilidad de los datos registrados se utilizan las pruebas que se proponen en trabajos como los de Castellano et al. (2000) y Gorospe et al. (2005).

Tras las sesiones de entrenamiento y formación se realizó una prueba de registro para el cálculo de la calidad del dato. La prueba de registro consistió en la clasificación de 37 tareas de entrenamiento correspondientes a tres sesiones de entrenamiento de la temporada 1 y otras tres sesiones de la 2. De esta prueba se obtuvieron resultados del entrenador (A 1), evaluador externo 1 (B 1) y evaluador externo 2 (C 1). Tres meses después, se procedió a realizar la misma prueba. En este caso, los resultados de la prueba 2 , serán presentados en referencia a los datos del entrenador (A 2), evaluador externo 1 (B 2) y evaluador externo 2 (C 2). Los registros realizados por el grupo de evaluadores y el entrenador de estudio, en el mismo período temporal, se utilizaron para calcular la fiabilidad interevaluador. Los registros realizados en dos momentos diferentes sirvieron para el cálculo de la fiabilidad intraevaluador.

Para determinar la calidad del dato y basados en los procedimientos utilizados en los trabajos de Castellano et al. (2000) y Gorospe et al. (2005) se realizaron dos tipos de pruebas: cálculo del índice de acuerdo y asociación utilizando el coeficiente de Pearson y la confiabilidad entre evaluadores a través del índice de kappa de Cohen y de multirater kfree.

Mediante el coeficiente de Pearson se evalúa en qué grado la frecuencia de cada categoría coinciden. Para el cálculo del coeficiente de Pearson se han utilizado las frecuencias absolutas de cada una de las categorías, de las variables del estudio, registradas en las dos pruebas. De esta forma se comprobó la fiabilidad interevaluador e intraevaluador. Los resultados del coeficiente de Pearson aparecen en la siguiente tabla: 
Estos resultados manifiestan la elevada fiabilidad tanto inter como intraevaluador. El cálculo de la calidad del dato, utilizando el índice de kappa de Cohen y de multirater kfree se realiza teniendo en cuenta el orden en que los evaluadores han registrado cada una de las categorías.

Para el cálculo de la fiabilidad intraevaluador (concordancia en dos medidas, un sólo evaluador) se utiliza el índice de kappa de Cohen (Martín, 2011).

Los valores de kappa de Cohen obtenidos en cada una de las variables son los siguientes: en la variable fase de juego la concordancia entre A1 y A2, B1 y B2 y C1 y C2 se manifiesta con un valor de .93 . En cuanto a la variable medio de entrenamiento, la concordancia entre A1 y A2 tiene un índice de Cohen de .70, de B1 y B2 de 0.80 y entre C1 y C2 es de .76. En lo que se refiere a la variable situaciones de juego A1 y A2 y $\mathrm{B} 1$ y $\mathrm{B} 2$ tienen un índice de .97 , mientras que el de $\mathrm{C} 1$ y C2 es de .93.

Para la fiabilidad interevaluador (tres evaluadores) se utiliza el índice de multirater kfree, una medida para los casos en los que existen más de dos evaluadores y estos no tienen establecidos a priori la distribución de los casos en cada una de las categorías (Randolph, 2005). En este caso se calculó la concordancia interevaluadores en las dos pruebas realizadas obteniendo los siguientes resultados: en la variable fases de juego se obtuvo un índice de .95 en ambas pruebas. En la variable medios de entrenamiento, en la primera prueba se obtuvo un índice de .77 y en la segunda de .60. En la variable situación de juego el valor de $k$ free en la primera prueba fue de .92 y en la segunda de .90 .

Los valores de la kfree en las variables situaciones de juego y fase de juego, reflejan que el grado de acuerdo es calificado de "casi perfecto". Aunque los valores de la variable medios de entrenamiento son calificados de un acuerdo "sustancial" con el objeto de mejorar la fiabilidad entre los mismos, se procedió a realizar un entrenamiento de los codificadores y una segunda prueba para el cálculo del valor de kappa. En este caso el valor de $k f$ ree fue igual a .88 lo que muestra un grado de acuerdo bastante elevado, calificado de "casi perfecto" (Landis y Koch, 1977).

Posteriormente, los datos introducidos por el entrenador fueron revisados por los dos evaluadores externos. Los datos que, a juicio de cada evaluador, eran "erróneos" fueron revisados en una sesión en la que estaban presentes los tres participantes en el proceso (entrenador y los dos evaluadores). En los casos en los que se precisara, los datos fueron modificados. Análisis estadístico

Se realizó un primer análisis descriptivo para conocer las características de la muestra estudiada y, posteriormente, un análisis inferencial para estudiar, si existen diferencias estadísticas en la variable de estudio entre ambas temporadas y, por otro lado, para relacionar y analizar las variables pedagógicas que describen el entrenamiento deportivo.

A través del programa informático, PyC Basket 2.0, y basados en el registro del tiempo que se dedica a cada una de las tareas de entrenamiento, se obtuvieron los porcentajes totales de cada una de las categorías de las fases de juego. La incorporación de los datos al paquete informático SPSS 15.0 permitió estudiar las diferencias estadísticamente significativas entre las dos temporadas analizadas y las relaciones con el resto de variables pedagógicas. La naturaleza de los datos, cualitativos, de tipo nominal, implica utilizar modelos matemáticos no paramétricos para el contraste de las hipótesis (Cubo, Martín y Ramos, 2011). Para estudiar las diferencias entre las temporadas se empleó el coeficiente de Wilcoxon. Con el objeto de profundizar en el tipo de situaciones de práctica que fueron planteadas para el tratamiento de cada una de las fases de juego, se estudió la asociación entre la variable fases de juego y las otras dos variables del estudio. Para ello, se empleó el coeficiente de contingencia. La interpretación de la asociación se realizó con los residuos tipificados corregidos $(R T C)$ de las tablas de contingencia (Ntoumanis, 2006; Williams y Wragg, 2006).

\section{Resultados y discusión}

En primer lugar se muestran los datos del análisis descriptivo de ambas temporadas continuando, posteriormente, con los del análisis inferencial.

Los resultados descriptivos se presentan de forma global (datos de toda la temporada deportiva) y pormenorizada, con datos de cada uno de los microciclos de las dos temporadas de estudio.

\section{Resultados por temporada}

La tabla 3 muestra los descriptivos de las categorías ataque y defensa en base al tiempo asignado a cada una de las tareas propuestas para cada una de las temporadas.

Tabla 3. Descriptivos de la variable fase de juego en base al tiempo asignado a cada una de las categorías de esta variable en las dos temporadas del estudio.

\begin{tabular}{|c|c|c|c|c|}
\hline \multirow{2}{*}{ FASES DE JUEGO } & \multicolumn{2}{|c|}{ Temporada 1} & \multicolumn{2}{|c|}{ Temporada 2} \\
\hline & Tiempo (min) & Porcentaje (\%) & Tiempo (min) & Porcentaje (\%) \\
\hline Ataque & 3030 & 70.55 & 1926 & 63.67 \\
\hline Defensa & 1265 & 29.45 & 1099 & 36.33 \\
\hline
\end{tabular}


La figura 1 muestra el número de tareas, expresado en porcentajes, dedicadas a cada una de las fases de juego en la tem-

Figura 1. Número de tareas dedicadas a cada una de las fases de juego en la temporada 1.

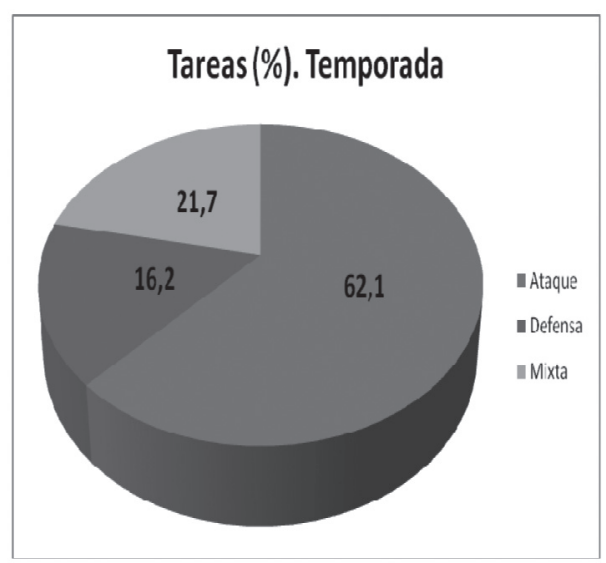

Tanto en el tiempo como en el número de tareas dedicadas a cada fase de juego, tabla 3 y figura 1 y 2 , se aprecia claramente el predominio del trabajo asignado al ataque por encima del de defensa en ambas temporadas.

Cañadas y García (2005) encuentran, tras analizar una temporada de un equipo de minibasket, que casi en un $50 \%$ de las actividades planteadas se trabajó la fase de ataque, siendo la fase mixta la segunda más tratada. Estos resultados, en sintonía con los datos del estudio de Moreira et al. (2007) de un equipo de categoría senior de baloncesto, muestran que en las sesiones de entrenamiento existe un predominio de la fase de ataque sobre la de defensa.

Existen discrepancias en cuanto a la importancia y tratamiento que se le debe dar a cada una de las fases de juego, o al menos en qué medida una debe ser superior a la otra. Sin embargo, la tónica general es conceder más importancia a la fase de ataque frente a la defensiva. Las opiniones de entrenadores (Cañadas, Ibáńez, et al., 2009; Ortega, 2010), jugadores (Palao et al., 2007) y expertos en este ámbito (Giménez y Sáenz-López, 2007; Ibáñez, 2000; Sáenz-López y Giménez, 2006) van en esta línea. porada 1, la figura 2 respecto a la temporada 2 .

Figura 2. Número de tareas dedicadas a cada una de las fases de juego en la temporada 2.

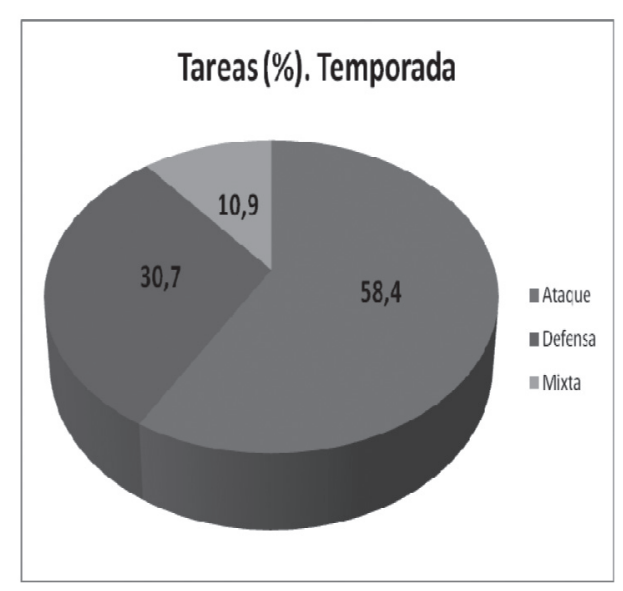

En trabajos como los de Palao et al. (2007) los resultados muestran que la motivación que despierta en los jugadores la fase de ataque es superior a la defensa. El hecho de que la fase de ataque sea más motivante puede implicar que en la iniciación se dedique más tiempo a esta fase de juego con el objeto de propiciar el aprendizaje y el gusto por este deporte (Giménez y Sáenz-López, 2007; Sáenz-López, 2009). Otra razón que se apunta para justificar el predominio de la fase de ataque frente a la defensa se debe a que este tipo de contenidos son más complejos y requieren de más tiempo de trabajo (Cańadas, Ibáñez, et al., 2009; Ortega, 2010; Sáenz-López y Giménez, 2006).

\section{Evolución a lo largo de la temporada}

A continuación se presentan, en la figuras 3 y 4, el tiempo asignado a cada fase de juego en cada uno de los microciclos de las temporadas de estudio. 
Figura 3. Tiempo asignado a cada fase de juego en cada uno de los microciclos de la temporada 1.

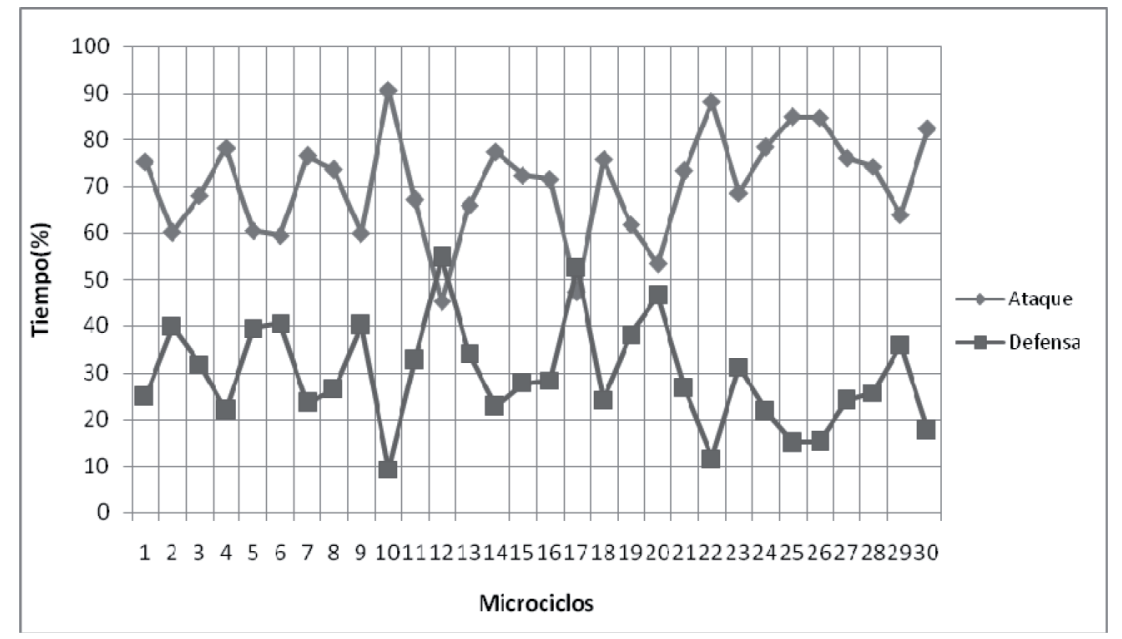

Figura 4. Tiempo asignado a cada fase de juego en cada uno de los microciclos de la temporada 2.

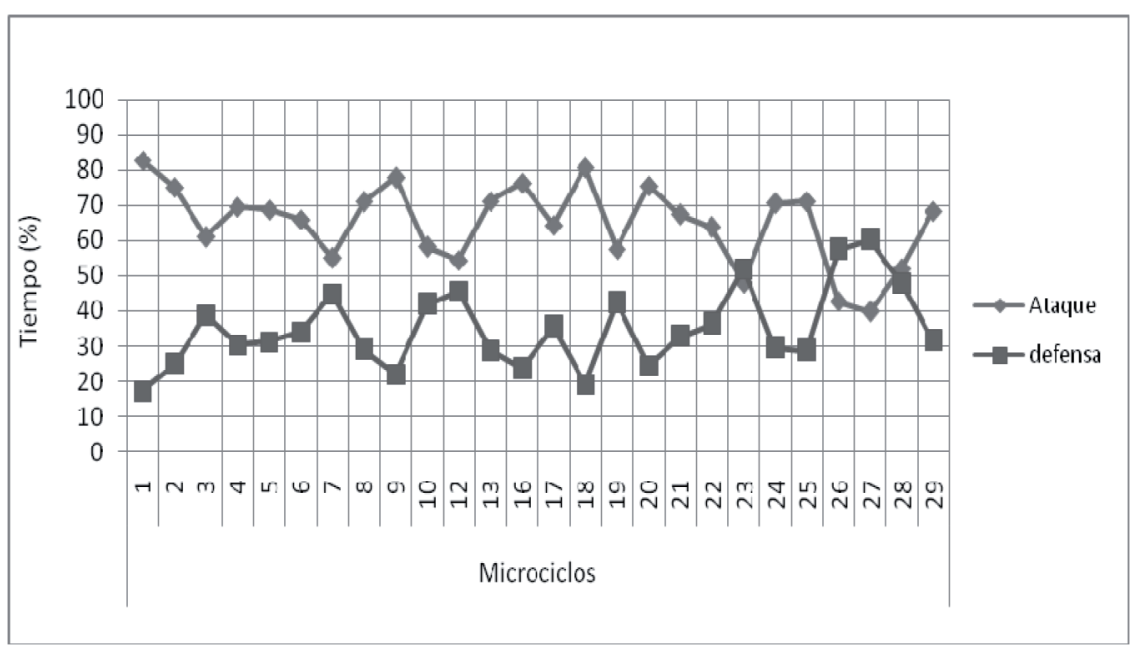

Tal y como apunta Ibáñez (2008) la importancia de controlar un aspecto como la fase de juego a lo largo de la temporada deportiva, va a proporcionar información de si se está desarrollando una formación equilibrada de los jugadores. Con el objeto de conseguir la formación completa del jugador, la tendencia es buscar un equilibrio desarrollando ambas fases (Sampedro, 1999).

La tendencia que muestran las gráficas están en sintonía con lo apuntado por Ibáñez (2008). Tal y como se puede apreciar en las figuras, existe una alternancia en los datos de la fase de ataque y defensa caracterizando un proceso ondulatorio.

Otra información que proporcionan estas figuras es ver cómo evoluciona el predominio de cada una de las fases a lo largo de la temporada. Aunque la tendencia ondulatoria sí que se mantiene a lo largo de toda la temporada, las líneas sólo llegan a cruzarse en ciertos microciclos, de forma que no se manifiesta el predominio de cada una de las fases de juego de forma alternativa. Sólo en dos ocasiones en la temporada 1 y en tres en la 2, se aprecia un predominio de la defensa sobre el ataque. Esto vuelve a confirmar el hecho de que, el trabajo dedicado al ataque suele ser superior al planificado para la defensa en cada una de las semanas de entrenamiento.

Aunque ya en los datos descriptivos se aprecian resultados similares en ambas temporadas del estudio se realiza un análisis inferencial para comprobar si existen diferencias mediante la prueba de Wilcoxon. Su resultado $(Z=-1.75, \mathrm{p}>.05)$ confirma la hipótesis de igualdad de los promedios comparados. Esto significa que no existen diferencias estadísticamente significativas en cuanto a las fases de juego tratadas en cada una de las temporadas del estudio. 
Relación entre las variables fase de juego $\mathrm{y}$ medios de entrenamiento

Tanto en la temporada $1\left(\chi_{(12, \mathrm{~N}=451)}^{2}=183.77, p<.001 ; C=.538\right.$, $p<.001)$, como en la $2\left(\chi_{(14, \mathrm{~N}=394)}^{2}=149.65, p<.001 ; C=.525\right.$, $p<.001)$ estas dos variables se relacionan estadísticamente de forma significativa manteniendo una asociación catalogada de media.

La situación que se describe en ambas temporadas es muy similar. Existe una proporción mayor de encontrar tareas en las que la fase de ataque se trabaje en situaciones analíticas, concretamente en un tipo de medios como son los ejercicios de aplicación simple (EAS) (temporada 1 RTC 5.7, temporada 2 RTC 4.5), y una proporción menor de lo esperado de hacerlo en situaciones lúdicas como el juego simple específico (JSE) (temporada $1 R T C-3.4$, temporada $2 R T C-2.5$ ). Sin embargo, la situación contraria se da en la fase de defensa, existe una proporción más alta de tareas enfocadas a los aspectos defensivos en los que se trabaja con JSE (temporada 1 $R T C$ 6.5, temporada $2 R T C 5.7$ ). En la temporada 1 el valor de los residuos tipificados corregidos no nos permite analizar con precisión la relación existente entre las categorías fase de defensa y EAS, sí en el caso de otra categoría de ejercicio como es el ejercicio de aplicación complejo (EAC) mostrando menos casos de los esperados en los que se produce esta asociación $(R T C-3.0)$. En la temporada 2 sí que se manifiesta que existen menos casos de los esperados en los que se utilicen los EAS para trabajar la defensa $(R T C-3.1)$.

En primer lugar surge la pregunta de ¿cómo es más adecuado trabajar los aspectos ofensivos y los defensivos? ¿De forma lúdica o analítica? Los resultados muestran que se ha planteado trabajar los fundamentos ofensivos con ejercicios y los defensivos sobretodo con juegos. Al respecto, no se encuentran referencias que distingan entre la forma de trabajar una fase de juego u otra y que nos aporten las posibles ventajas de utilizar medios como los juegos o los ejercicios con un tipo de contenido u otro. Sin embargo, y buscando posibles explicaciones a esta forma de plantear el trabajo de cada una de las fases de juego, si atendemos a las características de cada uno de estos medios de entrenamiento, una de las ventajas de la práctica analítica (ejercicios) es que reduce la complejidad de tareas o habilidades complejas. Sáenz-López y Giménez (2006) apuntan que los gestos técnicos de ataque son más difíciles de aprender que los de defensa, lo que puede explicar que, en mayor medida se trabajen los aspectos ofensivos en ejercicios. Otra posible razón puede deberse a la importancia concedida por el entrenador a cada una de las fases. Un menor interés por la fase defensiva puede ocasionar que no se persiga la perfección de gestos, no se haga tanto hincapié al respecto y por lo tanto no se trabaje de forma analítica.

La motivación que despierta cada fase de juego también puede influir de alguna forma. La motivación que despierta el ataque por encima de la defensa en los jugadores (Palao et al., 2007) puede llevar a utilizar medios de entrenamientos más motivantes como son los juegos (Giménez y Sáenz-López, 2007; Ibáñez, 2000; Sáenz-López, 2009) para contrarrestar esta diferencia.

\section{Relación entre las variables fase de juego y situación de juego}

Existe relación estadísticamente significativa entre estas dos variables tanto en la temporada $1\left(\chi^{2}{ }_{(24, N-451)}=287.1, p<.001\right.$; $C=.624, p<.001)$, como en la $2\left(\chi_{(18, \mathrm{~N}=394)}^{2}=86.69, p<.001\right.$; $C=.425, p<.001)$.

En ambas temporadas se plantea trabajar la fase de ataque, en mayor medida, con situaciones sin oposición del tipo $1 \mathrm{x} 0$ (temporada $1 R T C$ 9.7, temporada $2 R T C 5$ ) y $2 \mathrm{x} 0$ (temporada $1 R T C 5.1$, temporada $2 R T C$ 4.5). Mientras que para los contenidos defensivos se utilizan situaciones de $1 \mathrm{x} 1$ (temporada 1 RTC 12.3, temporada 2 RTC 4.9).

Para trabajar tanto los contenidos ofensivos como defensivos se han utilizado sobretodo situaciones de juego individuales mostrando, por un lado, el predominio de este tipo de situaciones de juego en los entrenamientos deportivos destinados a estas categorías. Estos resultados están en sintonía con otros trabajos como los de Fernandes y Tavares (2011) sobre un equipo de categoría superior 14-15 años y con las aportaciones de autores como Cárdenas (2006), entre otros, que opta por este tipo de situaciones para la primera etapa formativa de los jugadores de baloncesto.

Por otro lado, el hecho de que se opte por situaciones sin oposición para trabajar la fase de ataque, a modo de ejemplo un $42.9 \%$ de los contenidos ofensivos de la temporada 1 son tratados en situaciones de $1 \mathrm{x} 0$, manifiesta que existe un gran número de tareas en las que se practican los contenidos ofensivos en situaciones que se alejan de la situación de juego real. Es apuntando por varios autores el hecho de evitar las situaciones de práctica que se realizan sin oposición, aludiendo a las características de este deporte (Giménez y Sáenz-López, 2000; Ibáńez, 2000). El hecho de aprender y desarrollar los aspectos ofensivos en una práctica descontextualizada, alejada del juego real, puede generar problemas a la hora de aplicarlo posteriormente (Lorenzo y Prieto, 2002). Por un lado, la oposición supone un reto, aspecto que favorece el aprendizaje en estas edades (Sáenz-López, 2009), y por otro lado el defensor genera una incertidumbre respecto a la cual el jugador tiene que desarrollar la percepción y la toma de decisiones inherentes a este deporte. (Giménez y Sáenz-López, 2007; Ibáñez, 2000; Sáenz-López, 2009). Sin embargo, la realidad analizada en este trabajo muestra que se han propuesto menos tareas de las esperadas en las que se desarrollen el ataque con situaciones de $1 \mathrm{x} 1$ y por lo tanto sin oposición (temporada 1 RTC -10.7, temporada 2 RTC -6.0).

En lo que respecta a la fase de defensa, los resultados del 
estudio de Fernandes y Tavares (2011) en categoría cadete, están en sintonía con los de este trabajo. También encontraron que la situación de 1x1 fue más utilizada para trabajar aspectos defensivos que ofensivos.

De nuevo, la razón de que el entrenador le conceda menor importancia a este tipo de contenidos puede explicar el hecho de que el entrenador no haya querido "perder tiempo" trabajando en situaciones sin oposición y lo plante directamente en situaciones más reales. Por otro lado, el tipo de contenidos defensivos planteados puede que no tenga sentido trabajarlos sin la presencia de un oponente. Muchas de la acciones de la defensa son una reacción a las acciones del ataque (Carrillo y Rodríguez, 2009), si no existe el atacante puede resultar complejo que se produzcan las acciones de defensa que pretendemos trabajar.

Para la fase mixta se utilizan sobre todo situaciones de $2 \times 2$ (RTC 5.3), 4x4 (RTC 3.4) y 5x5 (RTC 3.7) en la temporada 1, mientras que es significativa en la temporada 2 la utilización de situaciones como el $1 \times 1$ ( $R T C 2.3)$, el $4 \times 4$ ( $R T C 2.1)$ y el $5 \times 5$ (RTC 2.5). Estos resultados muestran como el tratamiento de ambos tipos de fundamentos, ofensivos y defensivos, de forma indistinta sobretodo se plantean en situaciones de juego grupales o colectivas.

\section{Conclusión}

Se ha planificado dedicar un mayor número de tareas y asignar más tiempo al desarrollo de la fase de ataque frente a la defensa.

Se aprecia una tendencia ondulatoria que manifiesta la intención del entrenador por alternar el trabajo de ambas fases de juego a lo largo de toda la temporada deportiva.

El tratamiento de los contenidos ofensivos se plantea con la utilización de medios de entrenamiento como los ejercicios y con situaciones de juego sin oposición mientras que para el trabajo defensivo se opta por los juegos y las situaciones de $1 \mathrm{x} 1$.
No existen diferencias estadísticamente significativas entre ambas temporadas deportivas, lo que manifiesta que el planteamiento del trabajo de los fundamentos ofensivos y defensivos en ambas temporadas se sigue la misma línea.

En lo que respecta al entrenador del estudio, encargado del diseño del entrenamiento deportivo analizado, se puede definir una forma o modelo desde la que se plantea el tratamiento de las fases de juego en baloncesto que, en este caso, no se ha visto modificada de una temporada del estudio a otra. Las características de los jugadores, la experiencia y formación del entrenador (asociados al paso del tiempo), no parece haber influido en el entrenamiento de los fundamentos ofensivos y defensivos. En mayor medida, los resultados muestran que esta forma de entrenar se acerca a los principios que propone el modelo comprensivo basado en una metodología constructivista para la enseńanza del baloncesto. Sin embargo, existen aún muchos aspectos del entrenamiento sobre los que no hay referencias que puedan servir de guía a los entrenadores para plantear el entrenamiento deportivo desde esta perspectiva.

A través de este tipo de experiencia, basada en la planificación y registro del entrenamiento deportivo, se obtienen una serie de datos que, en primer lugar, proceden de la propia realidad, y en segundo lugar aportan una valiosa información sobre el entrenamiento deportivo que se desarrolla en categorías de iniciación.

Este tipo de trabajos se presenta como una opción bastante interesante para profundizar en el proceso de entrenamiento y así establecer las herramientas o recursos concretos para la optimización del entrenamiento deportivo. Este tipo de estudios puede ser aplicado a otros deportes, categorías, entrenadores con diferentes formación, características, etc., analizando cómo influyen cada uno de estos aspectos en los resultados del estudio. Todo ello con el objeto de acrecentar el conocimiento sobre el entrenamiento deportivo que repercutirá en la mejora de los proceso de formación del entrenador deportivo y, de forma directa, la de los jugadores.

\section{Referencias bibliográficas}

1. Cańadas, M., y García. J. (2005), Estudio de la metodología de entrenamiento utilizada en un equipo masculino de minibasket. En R. Martínez de Santos, S. Ibáńez y L. M. Sautu (Coords.), III Congreso Ibérico de Baloncesto. Libro de Actas (pp. 92). Vitoria-Gasteiz: AVAFIEP-FIEPZALEAK.

2. Cañadas, M., y Ibáńez, S. J. (2010). La planificación de los contenidos de entrenamiento de baloncesto en equipos de iniciación. E-Bm.com Revista de Ciencias del Deporte, 6(1), 49-65.

3. Cañadas, M., Ibáñez, S. J., García, J., y Parejo, I. (2009). Estudio del conocimiento pedagógico de los entrenadores de baloncesto que trabajan en etapas de formación. Habilidad motriz. Revista de Ciencias de la Actividad Física y del Deporte, 32, 44-52.

4. Cañadas, M., Parejo, I., Ibáńez, S. J., García, J., y Feu, S. (2009). Relación entre las variables pedagógicas de entrenamiento de un equipo de minibasket. Cuadernos de Psicología del Deporte, 9(Suppl.), 50.

5. Cárdenas, D. (2006, marzo). El proceso de formación táctica colectiva en el baloncesto desde la perspectiva constructivista. Lecturas Educación Física y Deportes, Revista Digital, 94. Recuperado el 22 de abril de 2008, de http://www.efdeportes.com/efd94/balonces.htm

6. Cárdenas, D. (2009). Bases para la construcción de una progresión didáctica. En G. Ortega y A. C. Jiménez (Coord.), Táctica y Técnica en la Iniciación al Baloncesto (pp. 197-216). Sevilla: Wanceulen

7. Carrillo, A., y Rodríguez, J. (2009). El desarrollo de los medios tácticotécnicos individuales y colectivos básicos de la fase de defensa en la iniciación al baloncesto. En G. Ortega y A. C. Jiménez (Coord.), Táctica y Técnica en la Iniciación al Baloncesto (pp. 19-56). Sevilla: Wanceulen

8. Castellano, J. Hernández, A., Gómez de Segura, P., Fontetxa, E., y Bueno, I. (2000). Sistema de codificación y análisis de la calidad del dato en el fútbol de rendimiento. Psicothema, 4(12), 635-641.

9. Cubo, S., Martín, B., y Ramos, J. L. (2011). Métodos de Investigación y Análisis de Datos en Ciencias Sociales y de la Salud. Madrid: Pirámide.

10. Fernandes, A., y Tavares, F. (2011). Descrição e análise dos conteúdos 
de treino numa equipa basquetebol de 14-15 anos, relativos a uma época desportiva. Revista portuguesa de ciências do desporto, 11 (Suppl. 4), 53.

11. Feu, S. (2006). El perfil de los entrenadores de balonmano. La formación como factor de cambio. Cáceres: Ilustre Colegio de Licenciados en Ciencias de la Actividad física y el Deporte.

12. García, J., Parejo, I., y Cañadas, M. (2010). Valoración de la carga de entrenamiento. Una experiencia real con un equipo de baloncesto de liga EBA. Revista Internacional de Deportes Colectivos, 5, 4-17.

13. Giménez, F. J., y Sáenz-López, P. (2007). Estrategias lúdicas para mejorar la percepción y decisión de los jugadores y jugadoras. En G. Ortega y A. C. Jiménez (Coord.), Baloncesto en la Iniciación (pp. 123-138). Sevilla: Wanceulen.

14. Gorospe, G., Hernández, A., Anguera, M. T., y Martínez de Santos, R. (2005). Desarrollo y optimización de una herramienta observacional en el tenis de individuales. Psicothema, 1(17), 123-127.

15. Graça, A. (1997). O Conhecimento Pedagógico do Conteúdo no Ensino do Basquetebol. Tesis doctoral no publicada. Faculdade de Ciências do Desporto e de Educação Física, Universidade do Porto, Oporto, Portugal.

16. Ibáńez, S. J. (2000). La enseńanza del baloncesto dentro del contexto educativo. Habilidad motriz. Revista de Ciencias de la Actividad Física y del Deporte, 15, 12-21.

17. Ibáńez, S. J. (2007). El control del entrenamiento como medio de formación permanente del entrenador de baloncesto. En S. J. Ibáńez, S. Feu, I., Parejo, J. García y M. Cañadas. (Eds.), IV Congreso Ibérico de Baloncesto. Desde la base a la élite deportiva. Libro de resúmenes y programa oficial (pp. 109). Cáceres: Copegraf.

18. Ibáńez, S. J. (2008). La planificación y el control del entrenamiento técnico-táctico en baloncesto. En N. Terrados y J. Calleja (Coord.), Fisiologia, entrenamiento y medicina del baloncesto (pp. 299-313). Barcelona: Paidotribo.

19. Ibáńez, S. J. (2009). La intervención del entrenador de baloncesto: investigación e implicaciones prácticas. En A. Lorenzo, S. J. Ibáńez y E. Ortega (Coords.), Aportaciones teóricas y prácticas para el baloncesto del futuro (pp. 11-30). Sevilla: Wanceulen Editorial Deportiva S. L.

20. Ibáñez, S. J., Macías, M., Pérez, M. A., y Feu, S. (2001). Planificación y control del entrenamiento en baloncesto. PYC-Basket, software aplicado. En S. J. Ibáñez y M. M. Macías (Eds.), Aportaciones al proceso de enseñanza y entrenamiento del baloncesto (pp. 193-201). Cáceres: Copegraf, s/l.

21. Ibáñez, S. J., Parra, M. A., y Asensio, J. M. (1999). Taxonomía de medios para la iniciación al baloncesto. Revista de entrenamiento deportivo, 13(4), 16-24.

22. Jiménez, F. (2000). Estudio praxiológico de la estructura de las situaciones de enseñanza en los deportes de cooperación/oposición de espacio común y participación simultánea: balonmano y fútbol sala. Tesis doctoral no publicada, Universidad de las Palmas de Gran Canaria, Las Palmas de Gran Canaria

23. Landis, J. R., y Koch, G. G. (1977). The measurement of observer agreement for categorical data. Biometrics, 33, 159-174.

24. Leite, N. (2009). Treino Desportivo em Basquetebol: Caracterização do processo de preparação desportiva a longo prazo em portugal. En A. Lorenzo, S. J. Ibáńez y E. Ortega (Coords.), Aportaciones teóricas y prácticas para el baloncesto del futuro (pp. 67-91). Sevilla: Wanceulen Editorial Deportiva S. L.

25. Lemos, I. (2005). O treinador. Conhecimento, Concepçóes e Prática. Estudo de Caso em Cinco Treinadores de Formação em Basquetebol. Dissertaçao para obtençao do grau de Mestre em Ciências do Desporto. Dissertação no publicada, Faculdade de Ciências do Desporto e de Educação Física, Universidade do Porto, Oporto, Portugal.
26. Lorenzo, A., y Prieto, G. (2002, mayo). Nuevas perspectivas en la enseñanza del baloncesto. Lecturas Educación Física y Deportes, Revista Digital, 48. Recuperado el 16 de febrero de 2005, de http://www.efdeportes.com/efd48/ensb.htm

27. Martín, B. (2011). Técnicas e instrumentos de recogida de investigación. En S. Cubo, B. Martín y J. L. Ramos (Coord.), Métodos de investigación $y$ análisis de datos en ciencias sociales y de la salud. Madrid: Ediciones Pirámide.

28. Martínez de Santos, R. (2004). La planificación del entrenamiento del baloncesto como estrategia mixta. En S. J. Ibáńez (Coord.), Cuadernos Técnicos. I Congreso Ibérico de Baloncesto. Monografía $N^{o} 1$ (pp. 32-39). Sevilla: Wanceulen.

29. Montero, I., y León, O. G. (2007). A guide for naming research studies in Psychology. International Journal of Clinical and Health Psychology, 7(3), 847-862.

30. Moreira, R., Pinto, D., y Graça, A. (2007). A periodização do treino de atletas seniores em basquetebol. Revista portuguesa de ciências do desporto, 7 (Suppl. 1), 21-84

31. Ntoumanis, N. (2006). A step-by-step guide to spss for sport and exercise studies. New York: Routledge.

32. Ortega, E. (2010). Medios técnico-tácticos colectivos en baloncesto en categorías de formación. Revista Internacional de Medicina y Ciencias de la Actividad Física y el Deporte, 38(10), 234-244.

33. Palao, J. M., Ortega, E., y Olmedilla, A. (2007). Technical and tactical preferentes among basketball players in formative years. Iberian Congress on Basketball Research, 4, 38-41.

34. Randolph, J. J. (octubre, 2005). Free-Marginal Multirater Kappa (multirater $\kappa$ free): An Alternative to Fleiss' Fixed-Marginal Multirater Kappa. Documento presentado en el Joensuu Learning and Instruction Symposium 2005, Universidad de Joensuu, Joensuu, Finlandia. En http://www. eric.ed.gov/PDFS/ED490661.pdf

35. Refoyo, I., Sampedro, J., Lorenzo, A., y del Campo, J. (2009). Valoración de las cargas de entrenamiento y competición. En F. Drobnic, J. Puigdellívol y T. Bové (Eds.), Bases cientificas para la salud y un óptimo rendimiento en Baloncesto (pp. 121-149). Madrid: Ergon.

36. Saad, M. A., y Nascimento, J. V. (2007). Estruturação das sessões de treinamento técnico-tático nos escalôes de formação do futsal. Revista portuguesa de ciências do desporto, 7(Suppl. 1), 21-84.

37. Sáenz-López, P. (2009). Diseńo de tareas tácticas y técnicas en la iniciación al baloncesto. En G. Ortega y A. C. Jiménez (Coord.), Táctica y Técnica en la Iniciación al Baloncesto (pp. 117-133). Sevilla: Wanceulen.

38. Sáenz-López, P., y Giménez, F. J. (2006). Metodología de enseñanza. En C. Torres (Coord.), La formación del educador deportivo en baloncesto. Bloque especifico Nivel II (pp. 51-78). Sevilla: Wanceulen.

39. Saenz-López, P, Ibáńez, S. J., Giménez, J., Sierra, A., y Sánchez, M (2005). Multifactor characteristic in the process of development of the male expert basketball player in Spain. International Journal of Sport Psychology, 36, 1-21.

40. Salado, J., Bazaco, M. J., Ortega, E., y Gómez, M. A. (2011). Opinión de los entrenadores sobre distribución de contenidos técnico-tácticos y pedagógicos en distintas categorías de baloncesto de formación. Cuadernos de Psicología del Deporte, 2, 51-62.

41. Sampedro, J. (1999). Fundamentos de Táctica Deportiva. Análisis de la estrategia de los deportes. Madrid: Gymnos.

42. Williams, C., y Wragg, C. (2006). Data analysis and research for sport and exercise science. A student guide. New York: Routledge. 